\title{
The New Atmospheric Scanning Electron Microscope allows in situ observation of
} dynamic phenomena under atmospheric pressure

\author{
M. Suga ${ }^{*}$, H. Nishiyama ${ }^{*}$, Y. Konyuba ${ }^{* *}$, Y. Watanabe ${ }^{* * *}$, S. Iwamatsu $^{* * *}$, D. Guarrera***, \\ C. Sato \\ *JEOL Ltd., 1-2 Musashino 3-chome, Akishima, Tokyo 196-8558, Japan \\ ** JEOL Engineering Ltd., 1-2 Musashino 3-chome, Akishima, Tokyo 196-8558, Japan \\ *** Yamagata Research Institute of Technology, Matsuei 2-2-1, Yamagata, 990-2473, Japan \\ **** JEOL USA Inc., 11 Dearborn Road, Peabody, MA 01960, USA \\ ${ }^{* * * * *}$ National Institute of Advanced Industrial Science and Technology, Tsukuba, 305-8566, Japan
}

Most chemical and physical reactions occur in liquid or gas. The new Atmospheric Scanning Electron Microscope (ASEM) is able to observe samples directly in liquid or gas under atmospheric pressure $[1,2]$. In this system, an electron-transparent window made of pressure-resistant 100nm-thick silicon nitride $(\mathrm{SiN})$ film, set into the bottom of the ASEM dish, allows an electron beam to be projected from underneath the sample (Fig. 1 a). Electrons backscattered from the sample are captured by a detector positioned below. Above the dish, an optical microscope (OM) realizes quasi-simultaneous observation. This combined system is called ClairScope (JASM-6200). We have also developed ASEM dishes with different functionalities to observe various phenomena in controlled environments.

The electrochemical ASEM dish has two gold/titanium bilayer electrodes $100 \mu \mathrm{m}$ apart, fabricated on the film using standard sputtering, photo-lithography, and wet etching techniques (Fig. $1 \mathrm{a}, \mathrm{b}$ ). Since the sample stage of the ASEM is open to the air, phenomena accompanying volume change can be observed, including electrochemical gas generation. These have not been easy to observe with the closed environmental cell. Using saturated $\mathrm{NaCl}$ solution as an electrolyte with $2.1 \mathrm{~V}$ applied between the electrodes, we observed electrochemical deposition near the cathode. Video was captured at a rate of 10 frames per second, showing tree-like structures growing toward the anode (Fig. 2).

The heated ASEM dish allows observation of temperature-dependent samples, using a heater and a thermocouple around the sample space (Fig. 3 a). A piece of solder (Sn: $42 \mathrm{wt} \%$, Bi: $58 \mathrm{wt} \%$ ) was placed on the SiN film and observed by ASEM at temperatures between 110 and 150 degrees Celsius (Fig. 3 b). Using the ASEM, we observed that the contrast from the segregated solder changed to uniform during melting (Fig $3 \mathrm{c}$, d) and the uniform contrast had various morphologies during solidification (Fig. 3 d, e, f).

\section{References}

[1] H. Nishiyama, et al., J. Struct. Biolog., (2010) In press.

[2] M. Suga, et al.,Microscopy and Microanalysis (2009), vol. 15, issue S2, p. 924. 


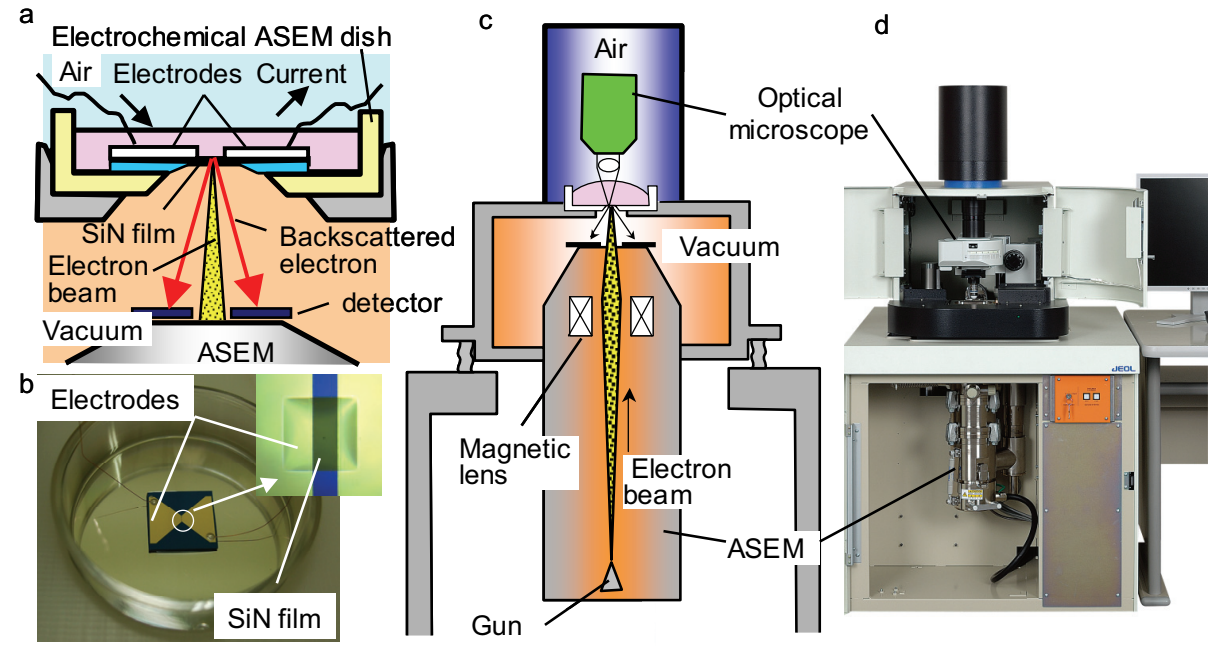

Fig. 1 (a) Cross-section and (b) photo of electrochemical ASEM dish, (c) cross-section and (d) photo of ClairScope, including the ASEM.

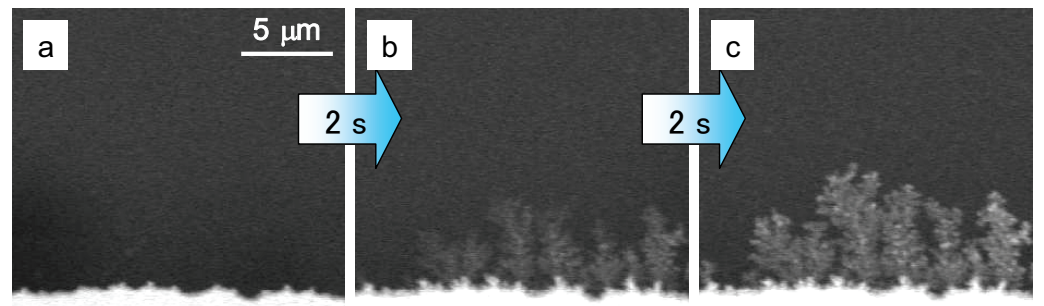

Fig. 2 Electrochemical deposition dynamically observed by ASEM near the cathode under current flow between the electrodes. The tree-like structure grew over time.

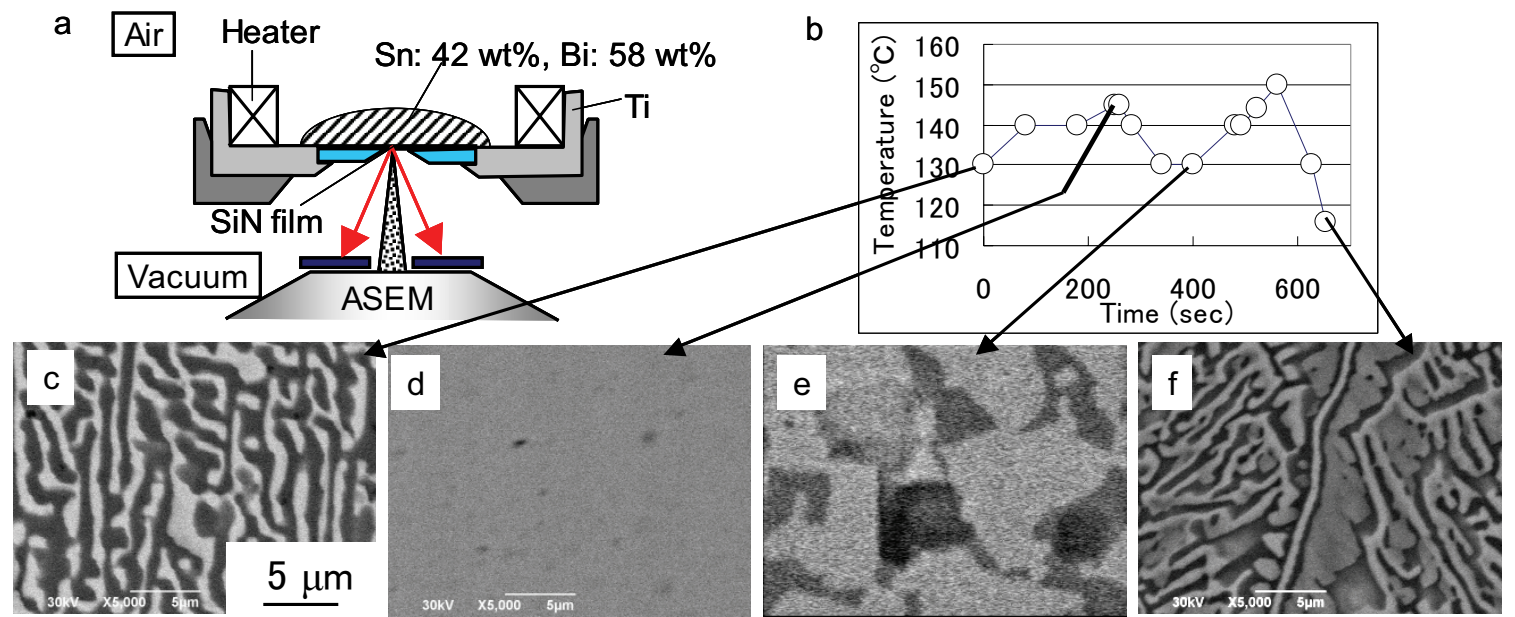

Fig. 3 (a) Cross-section of heated ASEM dish. (b) Temperature sequence dependence of (dynamic observation of) solder during heating and cooling. 